Santa Clara University

Scholar Commons

Biology

College of Arts \& Sciences

$10-1992$

\title{
Two mechanisms for growth inhibition by elevated transport of sugar phosphates in Escherichia coli
}

Robert J. Kadner

G. Patrick Murphy

Craig Stephens

SantaClara University, cstephens@scu.edu

Follow this and additional works at: http://scholarcommons.scu.edu/bio

Part of the Biology Commons

\section{Recommended Citation}

Kadner R. J., Murphy G. P., Stephens C. M. (1992). Two mechanisms for growth inhibition by elevated transport of sugar phosphates in Escherichia coli. J. Gen. Microbiol. 138, 2007-2014 10.1099/00221287-138-10-2007

This Article is brought to you for free and open access by the College of Arts \& Sciences at Scholar Commons. It has been accepted for inclusion in

Biology by an authorized administrator of Scholar Commons. For more information, please contact rscroggin@scu.edu. 


\title{
Two mechanisms for growth inhibition by elevated transport of sugar phosphates in Escherichia coli
}

\author{
Robert J. Kadner, ${ }^{*}$ G. Patrick Murphy and Craig M. Stephens $\dagger$ \\ Department of Microbiology, School of Medicine, University of Virginia, Charlottesville, VA 22908, USA
}

(Received 24 February 1992; revised 24 June 1992; accepted 2 July 1992)

\begin{abstract}
The Escherichia coli uhpT gene encodes an active transport system for sugar phosphates. When the uhpT gene was carried on a multicopy plasmid, amplified levels of transport activity occurred, and growth of these strains was inhibited upon the addition of various sugar phosphates. Two different mechanisms for this growth inhibition were distinguished. Exposure to glucose-6-phosphate, fructose-6-phosphate or mannose-6-phosphate, which enter directly into the glycolytic pathway, resulted in cessation of growth and substantial loss of viability. Cell killing was correlated with the production of the toxic metabolite, methylglyoxal. In contrast, addition of 2-deoxyglucose-6phosphate, galactose-6-phosphate, glucosamine-6-phosphate or arabinose-5-phosphate, which do not directly enter the glycolytic pathway, resulted in growth inhibition without engendering methylglyoxal production or cell death. Inhibition of growth could result from excessive accumulation of organophosphates in the cell or depletion of inorganic phosphate pools as a result of the sugar- $P / P_{i}$ exchange process catalysed by UhpT. The phosphatedependent uptake of glycerol-3-phosphate by the GlpT antiporter was strongly inhibited under conditions of elevated sugar-phosphate transport. There are thus two separate toxic effects of elevated sugar-phosphate transport, one of which was lethal and related to increased flux through glycolysis. It is likely that the control of uhp $T$ transcription by catabolite repression exists to limit the level of UhpT transport activity and thereby prevent the toxic events that result from elevated uptake of its substrates.
\end{abstract}

\section{Introduction}

The $u h p T$ gene of Escherichia coli encodes an active transport system for sugar phosphates (Weston \& Kadner, 1988). UhpT acts as an electroneutral phosphate antiporter to mediate the entry of a variety of organophosphates, including the phosphate esters of many sugars and sugar alcohols ranging in size from three to seven carbons, and glucosamine-6-phosphate (reviewed in Dietz, 1976; Maloney et al., 1990). The driving force for active transport of sugar phosphates is the outward movement of $P_{i}$ down the transmembrane $P_{i}$ gradient, which is normally maintained by the protonmotive-force-dependent Pit transport system (Sonna $e t$ $a l ., 1988)$. Production of the Uhp transporter is induced specifically by extracellular glucose-6-phosphate (Glu6P) or 2-deoxyglucose-6-phosphate (2dGlu6P) (Dietz \& Heppel, 1971) in a process mediated by the products of three regulatory genes, uhpABC (Weston \& Kadner, 1987). UhpA, which bears sequence relatedness

* Author for correspondence. Tel. (804) 924 2532; fax (804) 9821071.

† Current address : Department of Developmental Biology, Stanford University School of Medicine, Stanford, CA 94305, USA. to many transcriptional activators, including OmpR and NarL, appears to activate transcription of $u h p T$. Expression of UhpA from multicopy plasmids subverts the normal regulatory control by Glu6P and UhpBC function, and results in elevated and constitutive production of UhpT (Shattuck-Eidens \& Kadner, 1983).

Expression of UhpT is subject to catabolite repression and is reduced 2- to $3-$ fold when cells are grown in the presence of both Glu6P and glucose. This decrease is reversed by addition of cyclic AMP, and it has been found that the $u h p T$ promoter contains a typical binding site for the cyclic AMP receptor protein (CAP) (Merkel et al., 1992). Deletion of the CAP-binding site in the $u h p T$ promoter or of the $c r p$ gene resulted in an 8- to 10fold decrease in uhpT transcription and loss of the response to glucose. The susceptibility of $u h p T$ expression to catabolite repression was surprising. Glu6P itself is a potent catabolite repressor of unknown mechanism for numerous other systems (McGinnis \& Paigen, 1969) and it seems unlikely that its utilization should be repressed in preference for some other carbon source. A likely reason for the catabolite repression of $u h p T$ expression is to limit the amount of transport activity to 
prevent overloading the metabolic pathways with substrate. Ackerman et al. (1974) showed that the presence of Glu6P was lethal for wild-type $E$. coli when cyclic AMP was added to overcome catabolite repression. They showed that the toxicity was associated with elevated levels of methylglyoxal, as observed during deregulated metabolism of other carbon sources.

It was of interest to see whether similar toxicity could result from elevation of Uhp transport activity without interfering with catabolite repression. During studies of the genetic basis of $u h p T$ regulation, we observed that strains carrying $u h p T^{4}$ plasmids were sensitive to the presence of Glu6P in the medium. On solid media containing Glu6P, these strains grew poorly in the densely inoculated portion of streak plates but well as isolated colonies. Growth inhibition was even more dramatic in liquid media. This paper describes the deleterious effects of addition of sugar phosphates to strains overexpressing $u h p T$, and examines the genetic and environmental parameters influencing susceptibility to inhibition by sugar phosphates. Two distinctive effects of sugar phosphates are described: namely, lethal production of the toxic metabolite methylglyoxal due to overloading of the glycolytic pathway; and growth stasis elicited by sugar phosphates that are not directly metabolized through glycolysis. These results demonstrate the physiological importance of maintaining a limiting rate of transport during sugar phosphate catabolism and provide a clear rationale for the negative regulation of $u h p T$ expression by catabolite repression.

\section{Methods}

Bacterial strains. The Escherichia coli $\mathrm{K} 12$ strains were derived from strain RK4353 [ $\triangle($ argF-lac)U169 araD139 relA1 rpsL150 thi gyrA219 non]; strain RK 5000 also carried the $\triangle($ ilvB-uhpABCT)2056 deletion (Kadner \& Shattuck-Eidens, 1983). All host strains were recA56, unless otherwise specified. Plasmids were derived from pBR322. Plasmid pDSE1 carries a $19 \mathrm{~kb}$ insert from the Clarke-Carbon plasmid pLC1747 , which includes pColEl and the entire uhp region; pDSE6 carries the $9.5 \mathrm{~kb}$ insert from plasmid pLC40-33 and encodes only $u h p C$ and uhpT (Shattuck-Eidens \& Kadner, 1983). Plasmid pRJK 10 carries a $6.5 \mathrm{~kb}$ HindIII - BamHI fragment with a portion of the $i l v B N$ operon and the entire $u h p$ region (Weston \& Kadner, 1988). Four derivatives of pRJK 10 were made by insertion of a $1.5 \mathrm{~kb}$ kanamycin-resistance cassette into a restriction site in each of the four uhp genes.

Growth conditions. Cells were grown in MOPS medium (Neidhardt $e t$ al., 1974) or medium A (Davis \& Mingioli, 1950), with $0.5 \%$ glycerol as carbon source, thiamine $\left.(1 \mu \mathrm{g} \mathrm{ml})^{-1}\right)$, and required amino acids $(100 \mu \mathrm{g}$ $\mathrm{ml}^{-1}$ ). The nominal $\mathrm{P}_{\mathrm{i}}$ contents of MOPS medium and $\mathrm{A}$ medium are $1.3 \mathrm{mM}$ and $93.3 \mathrm{mM}$, respectively. When specified, casein hydrolysate (Difco) was added to $0.5 \%$. The salts media of different $\mathrm{pH}$ contained the same total concentration of phosphate and other ingredients as in medium $\mathrm{A}$, but were adjusted to the desired initial $\mathrm{pH}$ with $\mathrm{KOH}$. Ampicillin was added to $25 \mu \mathrm{g} \mathrm{ml}^{-1}$ to maintain plasmid presence. Cells were grown at $37^{\circ} \mathrm{C}$ with rotary shaking. Cell growth was measured as $\mathrm{OD}_{420}$. Viable cell counts were measured by dilution and plating on LB agar plates.

Measurement of methylglyoxal production. Production of a toxic factor by cells was detected by suspending cells in water at the time of addition of $300 \mu \mathrm{M}$-Glu6P. After $60 \mathrm{~min}$ at $37^{\circ} \mathrm{C}$, the cells were removed by centrifugation and the medium was passed through a $0.22 \mu \mathrm{m}$ filter and then concentrated by lyophilization. Serial dilutions of this material were pipetted onto a lawn of RK4353 on minimal agar plates, and zones of killing were observed after overnight incubation. Portions of the lethal extracts were incubated for $60 \mathrm{~min}$ with glutathione or glyoxylase I (Sigma) or both, and were then likewise pipetted onto RK4353 plates. The amount of $S$-lactyl-glutathione produced in the glyoxylase reaction was measured colorimetrically (Gawehn, 1983).

Methylglyoxal production and release from cells was measured by reaction of the growth medium with 2,4-dinitrophenylhydrazine (Cooper, 1975). Cells were incubated for various periods of time with sugar phosphates and the cells were removed by centrifugation. 2,4Dinitrophenylhydrazine $\left(665 \mu \mathrm{l}\right.$ of $0.25 \mathrm{mg} \mathrm{ml}^{-1}$ in $\left.2 \mathrm{M}-\mathrm{HCl}\right)$ was added to a $500 \mu \mathrm{l}$ sample containing various volumes of the growth medium $(2.5-500 \mu \mathrm{l})$. Following incubation for $15 \mathrm{~min}$ at $30^{\circ} \mathrm{C}, 835 \mu 110 \%$ (w/v) $\mathrm{NaOH}$ was added and $A_{550}$ determined. An absorbance of 16.4 corresponds to $1 \mu \mathrm{mol}$ methylglyoxal (Cooper, 1975). The chromatographic mobility of the 2,4-dinitrophenylhydrazone formed was compared to that derived from commercial methylglyoxal by ascending chromatography on dimethylformamide-soaked Whatman \#1 paper with a solvent system of $\mathrm{n}$-butyl ether/ethanol/water $(50: 5: 50)$.

Assay of transport activity. Transport of $\left[{ }^{14} \mathrm{C}\right]$-labelled glycerol-3phosphate or glucose-6-phosphate was measured as previously described (Shattuck-Eidens \& Kadner, 1981). Uptake is expressed in units of nmol substrate accumulated per $\mu$ l of cell water.

\section{Results}

\section{Effect of glucose-6-phosphate on cell growth and viability}

E. coli strains carrying plasmids pDSE1 or pRJK10, which contain the entire uhpABCT gene cluster with various extents of flanking sequence, exhibit high-level constitutive expression of $u h p T$. This amplification was shown by assays of Glu6P transport and of uhpT: :lacZ reporter fusions in vivo (Weston \& Kadner, 1988), and by determination of the level of Uhp transport activity following in vitro reconstitution into proteoliposomes (Sonna et al., 1988). When 3.5 mM-Glu6P was added to cultures of these strains in minimal salts/glycerol medium, growth was strongly inhibited after a short lag and viability began to decline within $30 \mathrm{~min}$ (Fig. $1 \mathrm{a}$ ). Cell killing proceeded with a half-time of about $12 \mathrm{~min}$ and led to a reduction in viable cell count by at least four orders of magnitude within $3 \mathrm{~h}$. Growth of the same host strain bearing the vector plasmid was unaffected by addition of Glu6P. The same cessation of growth and loss of viability was seen upon addition of $1.75 \mathrm{~mm}$-Glu6P (Fig. $1 b$ ), whereas $0.7 \mathrm{mM}$-Glu6P caused an inhibition of growth for 30-60 min followed by resumption at normal rate. 

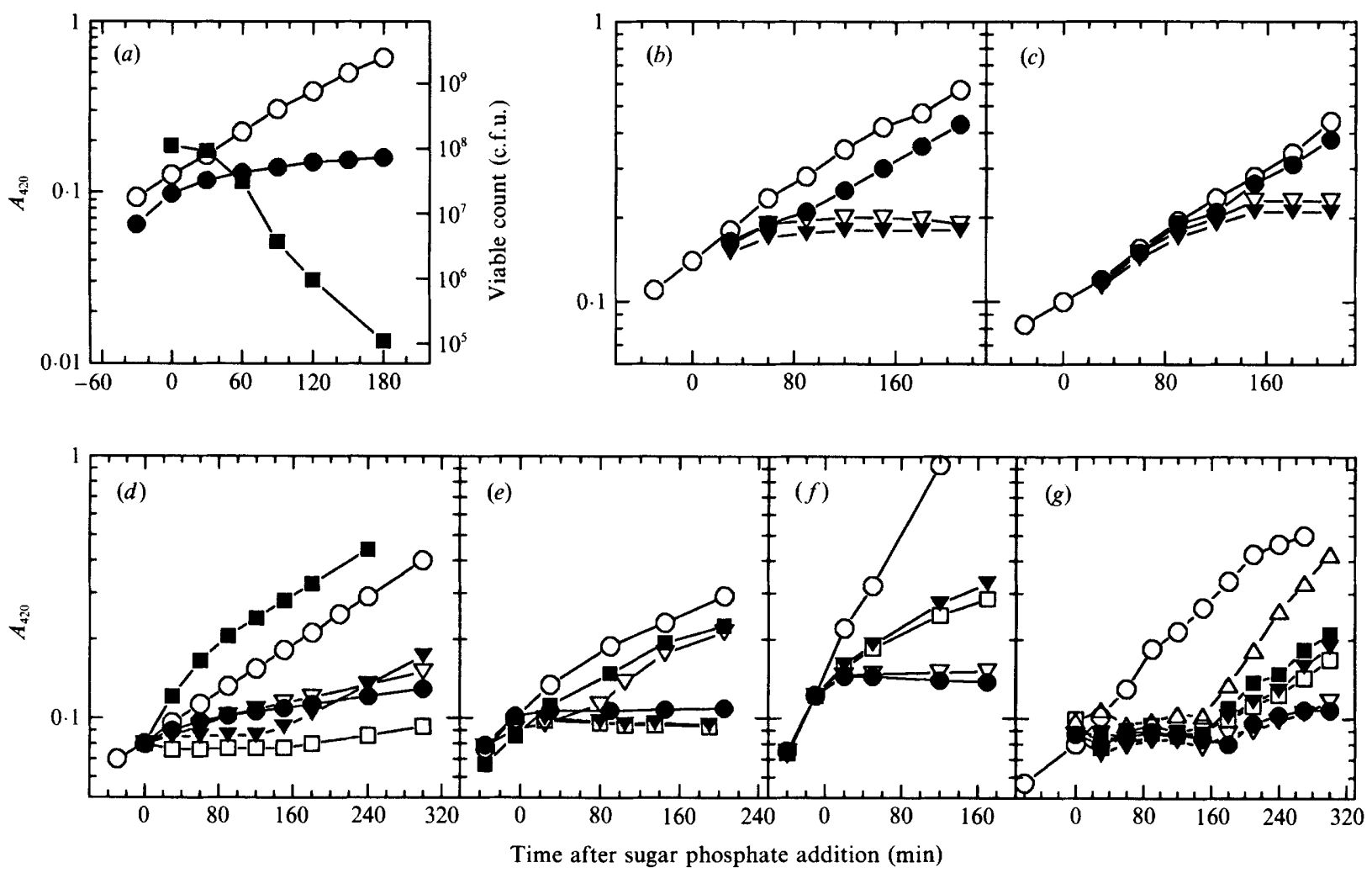

Fig. 1. Growth of $E$. coli strains carrying $u h p$ plasmids and exposed to sugar phosphates. Culture $\mathrm{OD}_{420}$ was measured at the indicated times after addition of the specified compounds at zero time. Experiments $(a-c)$ used medium A; $(d-g)$ used MOPS medium with $1.3 \mathrm{mM}-\mathrm{P}_{\mathrm{i}}$, unless otherwise specified. (a) Effect of Glu6P on growth and viability. Strain 4353 carrying pDSE1; turbidity after $O$, no addition or , $3.5 \mathrm{~mm}$-Glu6P addition; $\square$, colony-forming units after $3.5 \mathrm{~mm}$-Glu6P addition. (b) Effect of different concentrations of Glu6P. Strain RK4353 carrying pDSE1: $O$, control; $O$, Glu6P at $0.7 \mathrm{~mm} ; \triangle, 1.75 \mathrm{~mm}$; or $\nabla, 3.5 \mathrm{~mm}$. (c) Effect of Glu6P on an inducible strain. Strain RK4353 carrying pDSE6; symbols as (b). (d) Effect of different sugar phosphates. Strain RK5000 carrying pRJK10; O, control; $\odot$, Glu6P or Gal6P; $\nabla$, Fru6P; $\nabla$, glucosamine-6P; $\square$, 2-deoxyGlu6P; $\square$, ribose-5P; all sugar phosphates at $2.5 \mathrm{mM}$. (e) Effect of Gal6P on strains carrying transposon insertions in the $u h p$ plasmid. Strain RK 5000 carrying pRJK 10 with no addition $(\bigcirc)$ or with $2.5 \mathrm{mM}-\mathrm{Gal6P}(\Theta)$; or carrying a kanamycin resistance cassette in $u h p A(\nabla), u h p B(\nabla), u h p C(\square)$ or $u h p T(\square)$ and exposed to Gal6P. (f) Effect of $P_{i}$ growth medium concentration on response to 2.5 mM-Gal6P. Strain RK5000 carrying pRJK10 in MOPS medium; $O$, control; plus Gal6P in $P_{i}$ at $\bullet, 0.26 \mathrm{~mm} ; \nabla, 1.3 \mathrm{~mm} ; \nabla, 6.5 \mathrm{mM}$; or $\square, 13 \mathrm{mM}$. (g) Effect of growth medium on response to $2.5 \mathrm{~mm}$-Gal6P. Strain RK5000 carrying pRJK10 in MOPS medium; $O$, control; $0,2.5 \mathrm{~mm}-\mathrm{Glu} 6 \mathrm{P}$ in $1.3 \mathrm{mM}-\mathrm{P}_{\mathrm{i}} ; \nabla$, $2.5 \mathrm{~mm}-\mathrm{Gal} 6 \mathrm{P}$ in $1.3 \mathrm{~mm}-\mathrm{P}_{\mathrm{i}} ; \square, 2.5 \mathrm{mM}-\mathrm{Gal6P}$ in $2.6 \mathrm{~mm}-\mathrm{P}_{\mathrm{i}} ; \mathbf{\square}, 2.5 \mathrm{~mm}-\mathrm{Gal6P}$ in $7.8 \mathrm{~mm}-\mathrm{P}_{\mathrm{i}} ; \triangle, 2.5 \mathrm{~mm}-\mathrm{Gal} 6 \mathrm{P}$ in $1.3 \mathrm{~mm}-\mathrm{P}_{\mathrm{i}}$ grown with $0.25 \%$ glucose; $\nabla, 2.5 \mathrm{~mm}-\mathrm{Gal6P}$ in $1.3 \mathrm{~mm}-\mathrm{P}_{\mathrm{i}}$ grown with $0.25 \%$ galactose.

A different response to Glu6P was seen in cells carrying plasmid pDSE6, which contains $u h p T$ but not $u h p A$. Expression of the plasmid-borne $u h p T$ gene is dependent on the chromosomal copy of $u h p A$ and is inducible by Glu6P (Shattuck-Eidens \& Kadner, 1983). The addition of 1.75 or $3.5 \mathrm{~mm}$-Glu6P resulted in complete inhibition of growth (Fig. $1 c$ ) and the same degree of cell killing as in RK4353(pDSE1) (data not shown). However, there was a lag of 60-80 min before the onset of killing and a longer lag before the cessation of cell growth. The additional lag presumably represents the time required for induction of transporter expression to the level at which inhibitory levels of Glu6P accumulated in the cell. This view was supported by the effect of the inhibition of protein synthesis by chloram- phenicol (data not shown). Chloramphenicol (100 $\mu \mathrm{g}$ $\mathrm{ml}^{-1}$ ) caused a rapid cessation of growth in strains carrying either pDSE1 or pDSE6, but no loss of viability for at least $4 \mathrm{~h}$. Simultaneous addition of chloramphenicol and Glu6P ( $3.5 \mathrm{mM}$ ) to constitutively expressing strains carrying pRJK 10 or pDSE1 resulted in cell killing which was identical in extent and kinetics to that seen upon addition of Glu6P alone. In contrast, the inducible strain carrying pDSE6 was completely protected from killing by Glu6P when formation of the transport system was prevented by chloramphenicol. These results indicated that the observed lethality occurs in response to amplified transport of Glu6P, rather than to the amplified production of the transport protein itself. 
Table 1. Effect of sugar phosphates on growth, viability, and methylglyoxal production

Cells of strain RK 5000 (pRJK10) were grown in MOPS minimal medium with glycerol as carbon source and $1.32 \mathrm{mM}-\mathrm{P}_{\mathrm{i}}$. Sugars and sugar phosphates were added to $2.5 \mathrm{~mm}$, and after $120 \mathrm{~min}$, the relative change of culture turbidity or of colony-forming units was determined. Methylglyoxal was determined at this time as described in Methods.

\begin{tabular}{lccc}
\hline \hline \multicolumn{1}{c}{ Addition } & $\begin{array}{c}\text { Culture turbidity } \\
\text { (relative change) }\end{array}$ & $\begin{array}{c}\text { Viable counts } \\
\text { (relative change) }\end{array}$ & $\begin{array}{c}\text { Methylglyoxal } \\
\text { (mM) }\end{array}$ \\
\hline Control & 2.36 & 2.3 & 0.06 \\
Glucose-6P & 1.21 & 0.06 & 3.45 \\
Fructose-6P & 1.33 & 0.06 & 3.31 \\
Mannose-6P & 1.89 & 0.27 & 0.67 \\
Glucosamine-6P & 1.08 & 1.97 & 0.13 \\
2-Deoxyglucose-6P & $<1.05$ & 1.65 & 0.06 \\
Ribose-5P & 3.18 & 2.71 & 0.09 \\
Galactose-6P & $<1.05$ & $<1.15$ & 0.07 \\
Arabinose-5P & $<1.05$ & $<1.05$ & 0.06 \\
Glucose & 3.78 & ND & 0.06 \\
\hline \hline
\end{tabular}

ND, not determined.

\section{Response to other sugar phosphates}

The effect of other sugar phosphates on growth and viability was examined (Fig. $1 d$; Table 1). In RK5000(pRJK 10), 2.5 mM-fructose-6-phosphate (Fru6P) killed cells at the same rate and to the same extent as did Glu6P. Cells exposed to $2.5 \mathrm{~mm}$-mannose-6phosphate showed a similar increase in culture turbidity to the control culture, but there was a decrease in the number of colony-forming units that showed more daily variability and a lesser degree of killing than was seen with Glu6P or Fru6P. In contrast, 2-deoxyglucose-6phosphate (2dGlu6P) and galactose-6-phosphate (Gal6P) at $2.5 \mathrm{~mm}$ inhibited growth but did not cause cell death. Addition of $2.5 \mathrm{mM}$-glucosamine-6-phosphate stopped growth for about 120 min but with no apparent cell killing, and ribose-5-phosphate stimulated growth. Arabinose-5-phosphate also inhibited growth without cell killing. If the cells had been previously grown in the presence of arabinose, the growth inhibition by arabinose-5-phosphate was reduced or eliminated (data not shown). These results suggest that the effect on cell viability of the elevated transport of a sugar phosphate depends on the metabolic fate of that compound, such that lethality occurs only if the sugar phosphate enters the glycolytic pathway directly.

In the inducible strain RK4353(pDSE6), $3.5 \mathrm{~mm}$ Fru6P, which is a poor inducer of $u h p T$ expression, did not inhibit growth over the time period of this experiment (data not shown). 2-Deoxyglucose-6-phosphate is an effective inducer and caused a moderate growth inhibition but no killing. Arabinose-5-phosphate, which is not an inducer of the $u h p$ system, had no effect on this strain.

To determine the genetic requirements for this response to sugar phosphates, the effect of $2.5 \mathrm{mM}-\mathrm{Gal} 6 \mathrm{P}$ on uhpABCT deletion strain RK5000 containing pRJK 10 derivatives with mini-Tn 10 insertion mutations in each of the $u h p$ genes was examined. Gal6P caused growth inhibition in the $u h p B$ and $u h p C$ mutants, but not in strains with $u h p A$ or $u h p T$ insertions (Fig. 1e). Identical behaviour was seen in response to Glu6P, except that cell killing occurred along with growth inhibition (data not shown). The requirement for UhpA is consistent with previous observations that it is necessary for $u h p T$ expression, as transport activity is virtually undetectable in the absence of $u h p A$ even when $u h p T$ is in multicopy (Weston \& Kadner, 1988). When $u h p A$ is present on a multicopy plasmid, uhp $T$ expression in the absence of inducer is greatly increased and the $u h p B$ and $u h p C$ products are no longer necessary (Weston \& Kadner, 1988), consistent with the observation that Glu6P was lethal to $u h p B$ and $u h p C$ insertion mutants. Amplified expression of UhpT transport activity is clearly necessary for the toxic effect of sugar phosphates.

\section{Effect of medium conditions}

The effect of the composition of the growth medium on the response to Glu6P was examined. Considerable protection was provided by $0.5 \%$ casein hydrolysate at low Glu6P levels (data not shown). In its presence, $0.7 \mathrm{mM}-\mathrm{Glu} 6 \mathrm{P}$ did not inhibit growth to any obvious degree, and $1.75 \mathrm{~mm}$-Glu6P elicited only a $90 \mathrm{~min}$ lag, in contrast to the extensive killing in the absence of casein hydrolysate. The addition of $3.5 \mathrm{~mm}-\mathrm{Glu} 6 \mathrm{P}$ resulted in a similar degree of killing irrespective of the presence of casein hydrolysate. The protective effect could result from a reaction of amimo acids with a toxic agent generated upon metabolism of sugar phosphates.

A possible mechanism for the growth inhibition could be the acidification of the cytoplasm as a result of 


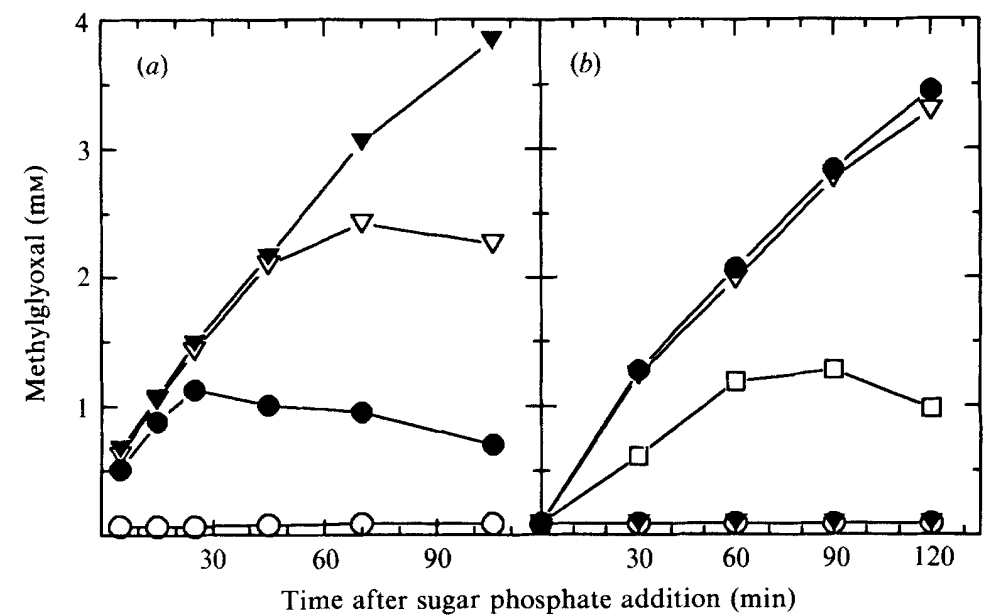

Fig. 2. Level of methylglyoxal in culture medium of strains exposed to sugar phosphates. The cell-free culture medium was obtained at the indicated times after addition of sugar phosphates and methylglyoxal measured by the 2,4-dinitrophenylhydrazine assay ( $1 \mu \mathrm{mol}=16.4 A_{550}$ units). Strain RK5000 carrying pRJK 10 was grown in MOPS medium. (a) Glu6P was added at $O, 0 \mathrm{~mm} ; 0,0.9 \mathrm{~mm} ; \nabla, 1.8 \mathrm{~mm}$; and $\boldsymbol{\nabla}, 3.5 \mathrm{~mm}$. These concentrations of Glu6P resulted in killing after $120 \mathrm{~min}$ of $82 \%, 97.3 \%$, and $97.5 \%$ of the cells. (b) Cells were exposed to sugar phosphates at $2.5 \mathrm{~mm}$ : $\bigcirc$, control; O, Glu6P; $\nabla$, Fru6P; $\square$, mannose-6P; or $\nabla$, any of glucosamine-6P, 2deoxyglucose-6P, Gal6P, or ribose-5P. elevated proton entry coupled directly or indirectly to uptake of sugar phosphates. However, the initial $\mathrm{pH}$ of the medium had only a slight effect of the susceptibility of strains to growth inhibition. The lag in growth that resulted after the addition of $0.7 \mathrm{~mm}-\mathrm{Glu} 6 \mathrm{P}$ was about 40 min longer at $\mathrm{pH} 5.5$ than at $\mathrm{pH} 7.5$, but growth was completely inhibited by $1.75 \mathrm{~mm}-\mathrm{Glu} 6 \mathrm{P}$ at both $\mathrm{pHs}$ (data not shown). The concentration of $P_{i}$ in MOPS medium had a significant effect on susceptibility to sugar phosphates. Increasing $\left[P_{i}\right]_{\text {out }}$ from $1.3 \mathrm{mM}$ to $6.8 \mathrm{mM}$ reduced the inhibitory effect of $2.5 \mathrm{mM}-$ Gal6P (Fig. $1 f$ ) and 2dGlu6P, but did not prevent the growth inhibition. Further increases to $13 \mathrm{mM}$ or $65 \mathrm{~mm}-\mathrm{P}_{\mathrm{i}}$ did not provide any additional protection. This result could indicate that external $P_{i}$ blocks entry of the growth-inhibitory sugar phosphates, or that higher external $P_{i}$ levels could partially overcome the depletion of internal $P_{i}$ pools by the action of the UhpT antiporter.

The presence of glucose in the medium blocked killing by Glu6P (data not shown) and reduced the inhibition caused by Gal6P (Fig. 1 $g$ ), most likely as a result of catabolite repression of $u h p T$ expression. Pregrowth of the cells with galactose did not relieve the inhibition caused by Gal6P.

\section{Formation of methylglyoxal}

Exposure of uhp-plasmid-bearing cells to Glu6P under conditions leading to lethality resulted in the production of a toxic factor which could be assayed by the killing of a lawn of sensitive cells. The toxic factor was inactivated by incubation with glyoxylase I plus glutathione but not with either alone. This result suggested that the toxic factor was a ketoaldehyde, most likely methylglyoxal (MG). In support of this proposal, cells exposed to Glu6P released material reactive with 2,4-dinitrophenylhydrazine (DNPH). The derivative formed with the growth medium of Glu6P-treated cells was identical in absorp- tion spectrum and chromatographic mobility with the corresponding derivative formed with commercial $\mathrm{MG}$ (data not shown). MG has been shown to accumulate in cells in which the metabolic flux through the glycolytic pathway has been increased through some mechanism to subvert the usual regulation of metabolism (reviewed in Cooper, 1984).

To demonstrate the linkage between high-level sugar phosphate transport, MG production and cell death, MG levels in cultures were measured at various times after addition of Glu6P. MG levels rose rapidly after Glu6P addition, and the amount of $\mathrm{MG}$ produced was related to the concentration of Glu6P added (Fig. 2a). Even non-lethal concentrations of Glu6P engendered MG excretion, but the levels in the medium decreased to a basal level, at which time growth resumed. Incubation with $3.5 \mathrm{~mm}-$ Fru6P resulted in the production of $\mathrm{MG}$ at the same rate and extent as in cells incubated with Glu6P (Fig. 2b; Table 1). In contrast, there was no detectable synthesis of MG upon incubation with sugar phosphates (2dGlu6P, Gal6P, glucosamine-6-phosphate, or ribose-5phosphate) that inhibited growth but did not cause killing (Table 1). Thus, cell killing following amplified uptake of some sugar phosphates was correlated with the formation of $\mathrm{MG}$.

\section{Function of GlpT under conditions of high-level UhpT activity}

The basis for the growth inhibition resulting from excessive transport of the sugar phosphates that did not engender MG production is less obvious. It was possible that excessive transport of sugar phosphates resulted in depletion of the intracellular $P_{i}$ pool to a growth-limiting level. To examine this possibility, the transport of $\left[{ }^{14} \mathrm{C}\right]$ glycerol-3-phosphate by GlpT, a phosphate antiporter related in sequence and mechanism to UhpT (Eiglmeier et al., 1987), was examined with or without 


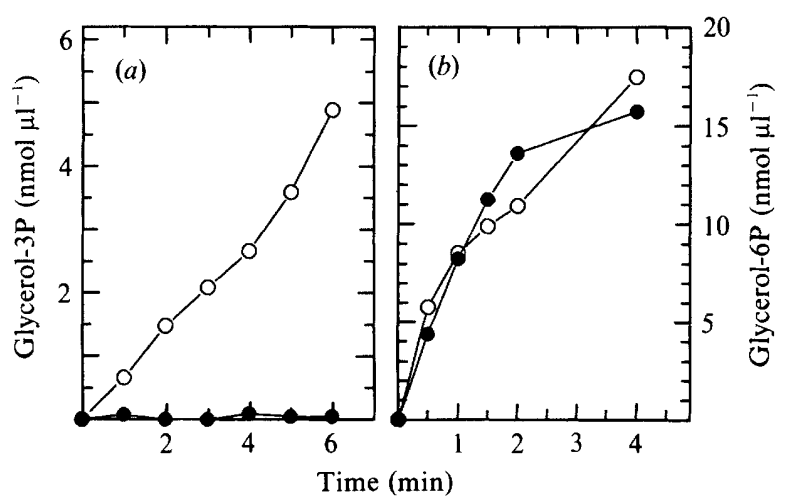

Fig. 3. Effect of 2deoxyGlu6 $P$ on glycerol-3P uptake and of $P_{i}$ on Glu6P uptake. Cells of strain RK 5000 carrying pRJK 10 were grown in MOPS/glycerol medium. (a) To one of duplicate cultures was added 2deoxyGlu6P to $2.5 \mathrm{~mm}$ for $88 \mathrm{~min}$ before assay of uptake of $\left[{ }^{14} \mathrm{C}\right.$ ]glycerol-3P $\left(200 \mu \mathrm{M} ; 0 \cdot 2 \mu \mathrm{Ci} \mathrm{ml}^{-1}\right) ; \bigcirc$, no addition;, $2 \mathrm{dGlu} 6 \mathrm{P}$. (b) Cells grown in MOPS/glycerol medium with $\mathrm{P}_{\mathrm{i}}$ concentrations of $\mathrm{O}$, $1.3 \mathrm{~mm}$ or, $26 \mathrm{~mm}$. Uptake of $\left[{ }^{14} \mathrm{C}\right] \mathrm{Glu} 6 \mathrm{P}\left(200 \mu \mathrm{M} ; 0.2 \mu \mathrm{Ci} \mathrm{ml}^{-1}\right)$ was measured.

exposure of cells to Gal6P or 2dGlu6P. Transport of glycerol-3-phosphate was strongly inhibited by addition of 2dGlu6P (Fig. 3a); similar inhibition was seen upon addition of Gal6P (data not shown). Inhibition of transport occurred within $10 \mathrm{~min}$ after addition of the hexose phosphate and was relieved when cells resumed growth following prolonged incubation (data not shown).

The partial reduction in growth inhibition that results from increased $\mathrm{P}_{\mathrm{i}}$ in the growth medium (Fig. $1 f$ ) was also consistent with the view that amplified sugarphosphate transport might deplete intracellular $P_{i}$ pools. However, it was possible that high external $P_{i}$ might competitively inhibit sugar phosphate uptake and thereby relieve the growth inhibition by decreasing the accumulation of sugar phosphate in the cell. To investigate this explanation, the rate of uptake of $\left[{ }^{14} \mathrm{C}\right]$ Glu6P was measured in media with $1.3 \mathrm{~mm}$ and 26 mM- $P_{i}$ (Fig. $3 b$ ). High external $P_{i}$ had only a slight effect if any on the rate of Glu6P uptake under these conditions. These results are consistent with the proposal that the nonlethal inhibitory effect of amplified sugar phosphate transport is dependent on the accumulation of the internal sugar phosphate, acting by depletion of the internal $P_{i}$ pool or accumulation of osmotically active, charged metabolites.

\section{Discussion}

The results presented here show that elevated transport of sugar phosphates in E. coli strains overproducing the UhpT hexose-phosphate transport protein is deleterious to the cell by at least two mechanisms. Some sugar phosphates caused extensive cell killing, whereas others resulted only in growth inhibition. Lethality was correlated with the ability of the transported sugar phosphate to enter the glycolytic pathway of metabolism and to engender MG synthesis. Glu6P and Fru6P are intermediates in glycolysis and rapidly caused a drastic drop in cell viability. In contrast, 2dGlu6P, which cannot be converted to Fru6P and is not metabolized further, and other sugar phosphates (galactose-, arabinose-, and ribose-phosphates), which are utilized by alternative catabolic pathways, did not lead to MG synthesis and were not lethal.

Previous studies have shown that elevated metabolic fluxes through the glycolytic pathway can engender cell killing by generating excessive levels of methylglyoxal (Freedberg et al., 1971; Ackerman et al., 1974). These abnormally high metabolic rates were achieved in two ways. Growth of wild-type cells in the presence of an inducing sugar, such as xylose or Glu6P, and cyclic AMP overcame catabolite repression and resulted in high level synthesis of the corresponding transport system (Ackerman et al., 1974). High rates of glycerol metabolism required use of mutant strains which both expressed the glycerol-catabolic enzymes at a high, constitutive level, owing to loss of the GlpR repressor, and also possessed an altered glycerol kinase activity that was insensitive to feedback inhibition by fructose-1,6-bis-phosphate (Freedberg et al., 1971). Amplified copy number of the uhpT gene provides another mechanism to increase glycolytic flux. MG production can explain some of the curious phenotypic properties of strains carrying Uhp plasmids. Growth on agar plates containing sugar phosphates as sole carbon source was healthy in regions of lower inoculum size but was sparse in the regions of high cell density, and growth in liquid medium in the presence of Glu6P was very poor. The growth of isolated colonies is probably explained by the diffusion of $\mathrm{MG}$ away from cells into the agar medium; at high cell density in liquid or solid medium, MG accumulates to toxic concentrations.

MG is formed from dihydroxyacetone phosphate by the enzyme methylglyoxal synthase and is converted to lactate by glyoxylase $I$ in the presence of glutathione (reviewed in Cooper, 1984). A physiological role for MG synthesis has yet to be firmly established, however, MG toxicity in $E$. coli has been extensively documented. Toxicity appears to result primarily from inhibition of protein synthesis, though DNA synthesis may also be affected (Fraval \& McBrien, 1980). It appears from this and previous work (Freedberg et al., 1971; Ackerman et al., 1974) that 'proper' regulation of metabolic flow through glycolysis (i.e. that which is sufficient to avoid overproduction of $\mathrm{MG}$ ) is dependent on the rate of entry of substrates into the pathway being limited at the 
transport step, and can be subverted by elevated transport activities. It should also be noted that the potentially low intracellular levels of $P_{i}$ developed during Glu6P/Fru6P transport as a result of the phosphate antiport activity of UhpT would also favor MG production as $M G$ synthase is inhibited by $P_{i}$ and the alternate, normal route of glycolytic formation of glyceraldehyde-3-phosphate is $\mathrm{P}_{\mathrm{i}}$-dependent.

The observation that mannose-6-phosphate resulted in lower MG production and less cell death than seen with Glu6P and Fru6P, while glucosamine-6-phosphate resulted only in transient growth inhibition is interesting, considering that both sugars can be enzymically converted into Fru6P, and thus could potentially enter glycolysis. Both compounds also have biosynthetic roles, with mannose-6-phosphate being involved in capsular polysaccharide synthesis and glucosamine-6-phosphate in peptidoglycan and LPS synthesis. It is possible that glucosamine-6-phosphate is shunted preferentially into the synthetic pathway, which is essential for cell growth, rather than the dissimilatory pathway. Capsule synthesis, in contrast, is a dispensable function which may not be expressed under the growth conditions employed here. The delayed effect of mannose-6-phosphate may have been due to limiting levels of phosphomannoisomerase, the enzyme which converts mannose-6-phosphate into Fru6P.

The physiological significance of the regulation of uhpT expression by catabolite repression must be to reduce the flux of sugar phosphate transport to limiting levels. Rates of transport must be limited because the feedback controls of glycolytic activity are obviously not sufficient to avoid deleterious consequences of elevated substrate presentation. The detrimental effects resulting from excessive transport cannot be ascribed solely to toxic levels of MG arising from excessive production of dihydroxyacetone phosphate. Substrates that do not elicit $\mathrm{MG}$ production still caused very significant growth inhibition. This inhibition could be related to the very familiar growth inhibition caused by the accumulation of sugar phosphates in mutant strains that are blocked in their further metabolism. The basis for this effect is not clear, but might involve a salting-out effect owing to the sequestration of intracellular water by the high levels of organophosphates $(>10 \mathrm{~mm})$.

The fact that increased transport of sugar phosphates strongly inhibited glycerol-3-phosphate transport is consistent with a depletion of the internal $P_{i}$ pool by the antiport action of UhpT. This model is consistent with the protective effect of increased $\left[\mathrm{P}_{\mathrm{i}}\right]_{\text {out }}$ against the addition of Gal6P or 2dGlu6P. This protective effect of higher $\left[\mathrm{P}_{\mathrm{i}}\right]_{\text {out }}$ was not due simply to competition of $\mathrm{P}_{\mathrm{i}}$ with Glu6P for entry on UhpT, since the directly measured rate of Glu6P uptake was not significantly inhibited at this concentration of external $\mathrm{P}_{\mathrm{i}}$. Assuming that the $K_{\mathrm{m}}$ for $P_{i}$ for GlpT is similar to that for UhpT (1.2 mM), it is likely that the level of internal $P_{i}$ could be reduced to a level low enough to affect antiporter activity. Consistent with this view is the puzzling finding that the level of Glu6P uptake in uhpT-plasmid-bearing cells is only increased 2-4 fold over that of an induced haploid strain, despite the fact that reconstitution assays showed that the amount of active UhpT protein was increased more than 20-fold (Sonna et al., 1988). This discrepancy may be explained by the suggestion that sugar phosphate transport in whole cells is not limited by the number of transporters, but by some physiological factor, potentially the capacity of the $P_{i}$ pool. The inhibition of GlpT by UhpT substrates is consistent with this view. However, given the $K_{\mathrm{m}}$ values observed for UhpT, it is unlikely that the level of intracellular $\mathrm{P}_{\mathrm{i}}$ could be reduced by the action of UhpT to below $5 \mu \mathrm{M}$, at which growth inhibition occurs. Thus, the mechanism for inhibition of growth by accumulated sugar phosphates remains to be defined. These findings provide evidence for multiple mechanisms of growth inhibition by elevated rates of carbohydrate transport and reinforce the need for careful regulation of transport activity as a primary site of metabolic control.

This work was supported by research grant GM38681 from the National Institute of General Medical Sciences.

\section{References}

Ackerman, R. S., Cozzarelli, N. R. \& Epstein, W. (1974). Accumulation of toxic concentrations of methylglyoxal by wild-type Escherichia coli K-12. Journal of Bacteriology 119, 357-362.

COOPER, R. A. (1975). Methylglyoxal synthase. Methods in Enzymology 41, 502-508

COOPER, R. A. (1984). Metabolism of methylglyoxal in microorganisms. Annual Review of Microbiology 38, 49-68.

DAvis, B. D. \& Mingioli, E. S. (1950). Mutants of Escherichia coli requiring methionine or vitamin $B_{12}$. Journal of Bacteriology $60,17-$ 28.

DieTz, G. W. (1976). The hexose phosphate transport system of Escherichia coli. Advances in Enzymology 44, 237-259.

DieTZ, G. W. \& HePpel, L. A. (1971). Studies on the uptake of hexose phosphates. II. The induction of the glucose-6-phosphate transport system by exogenous but not by endogenously formed glucose-6phosphate. Journal of Biological Chemistry 246, 2885-2890.

EIGLMeIER, K., Boos, W. \& Cole, S. T. (1987). Nucleotide sequence and transcriptional start point of the glpT gene of Escherichia coli: extensive sequence homology of the glycerol-3-phosphate transport protein with components of the hexose-6-phosphate transport system. Molecular Microbiology 1, 251-258.

Fraval, H. N. A. \& McBrien, D. C. H. (1980). The effect of methyl glyoxal on cell division and the synthesis of protein and DNA in synchronous and asynchronous cultures of Escherichia coli $\mathrm{B} / \mathrm{r}$. Journal of General Microbiology 117, 127-134.

FreedberG, W. B., Kistler, W. S. \& Lin, E. C. C. (1971). Lethal synthesis of methylglyoxal by Escherichia coli during unregulated glycerol metabolism. Journal of Bacteriology 108, 137-144.

GAWEHN, K. (1983). Methylglyoxal. In Methods of Enzymatic Analysis, vol. 6, pp. 593-596. Edited by H.-U. Bergmeyer. Weinheim: Verlag Chemie. 
KadNer, R. J. \& Shattuck-EIdens, D. M. (1983). Genetic control of the hexose phosphate transport system of Escherichia coli: mapping of deletion and insertion mutations in the uho region. Journal of Bacteriology 155, 1052-1061.

McGinnis, J. F. \& Paigen, K. (1969). Catabolite inhibition: a general phenomenon in the control of carbohydrate utilization. Journal of Bacteriology 100, 902-913.

Maloney, P. C., Ambudkar, S. V., Anantharam, V., Sonna, L. A. \& VARADHACHARY, A. (1990). Anion-exchange mechanisms in bacteria. Microbiological Reviews 54, 1-17.

Merkel, T. J., Nelson, D. M., Brauer, C. L. \& KadNer, R. J. (1992). Promoter elements required for positive control of transcription of the Escherichia coli uhpT gene. Journal of Bacteriology 174, 27632770 .

Neidhardt, F. C., Bloch, P. L. \& Smith, D. F. (1974). Culture medium for enterobacteria. Journal of Bacteriology 119, 736-747.

ShatTuCK-EIdENS, D. M. \& KADNER, R. J. (1981). Exogenous induction of the Escherichia coli hexose phosphate transport system defined by uhp-lac operon fusions. Journal of Bacteriology 148, 203 209.

Shattuck-EIdens, D. M. \& KadNER, R. J. (1983). Molecular cloning of the $u h p$ region and evidence for a positive activator for expression of the hexose phosphate transport system of Escherichia coli. Journal of Bacteriology 155, 1060-1070.

Sonna, L. A., Ambudkar, S. V. \& Maloney, P. C. (1988). The mechanism of glucose 6-phosphate transport by Escherichia coli. Journal of Biological Chemistry 263, 6625-6630.

WESTON, L. A. \& KADNER, R. J. (1987). Identification of Uhp polypeptides and evidence for their role in exogenous induction of the sugar phosphate transport system of Escherichia coli. Journal of Bacteriology 169, 3546-3555.

WESTON, L. A. \& KADNER, R. J. (1988). Role of uhp genes in expression of the Escherichia coli sugar-phosphate transport system. Journal of Bacteriology 170, 3375-3383. 